\title{
Asymptotic dimension of discrete groups
}

\author{
by
}

A. Dranishnikov and J. Smith (Gainesville, FL)

\begin{abstract}
We extend Gromov's notion of asymptotic dimension of finitely generated groups to all discrete groups. In particular, we extend the Hurewicz type theorem proven in [B-D2] to general groups. Then we use this extension to prove a formula for the asymptotic dimension of finitely generated solvable groups in terms of their Hirsch length.
\end{abstract}

1. Introduction. The main purpose of this paper is to extend the formula $\operatorname{asdim} \Gamma \leq h(\Gamma)$ from [B-D2], which gives an upper bound for the asymptotic dimension of nilpotent finitely generated groups in terms of the Hirsch length, to solvable groups. The main problem here is that even working with finitely generated groups one has to consider infinitely generated ones. This is because a subgroup of a finitely presented group can be infinitely generated. Originally, asymptotic dimension was defined only for finitely generated groups $[\mathrm{Gr}]$. It was actually defined as a quasi-isometry invariant of metric spaces. Since all word metrics induced by a finite set of generators in a finitely generated group are quasi-isometric, the asymptotic dimension gives a group invariant. It turns out that the asymptotic dimension of a finitely generated group is a coarse invariant (definition below) $[\mathrm{Ro} 2]$.

In this paper we notice that every countable group admits a left invariant proper metric and any two such metrics are coarsely equivalent. Thus, one can extend the notion of asymptotic dimension to all countable groups. We show in $\S 2$ that the Hurewicz type formula of [B-D2] still holds for countable groups. As a result we prove the asymptotic dimension formula for solvable groups $(\S 3)$. The main ingredient of $\S 2$ is Theorem 2.1, which states that the asymptotic dimension of a countable group $G$ is the maximum of the asymptotic dimensions of the finitely generated subgroups. This rule leads to a definition of the asymptotic dimension for an arbitrary group $(\S 2)$.

2000 Mathematics Subject Classification: Primary 20F69.

Key words and phrases: asymptotic dimension, solvable group, Hirsch length.

The first author was partially supported by NSF grants DMS-0305152. 
We recall that a map $f:(X, d) \rightarrow\left(Y, d^{\prime}\right)$ between metric spaces is a coarse embedding if there are functions $\varrho_{1}, \varrho_{2}: \mathbb{R}_{+} \rightarrow \mathbb{R}_{+}$tending to infinity such that

$$
\varrho_{1}\left(d\left(x, x^{\prime}\right)\right) \leq d^{\prime}\left(f(x), f\left(x^{\prime}\right)\right) \leq \varrho_{2}\left(d\left(x, x^{\prime}\right)\right)
$$

for all $x, x^{\prime} \in X$. A coarse embedding $f:(X, d) \rightarrow\left(Y, d^{\prime}\right)$ is a coarse equivalence if the image $f(X)$ is $R$-dense in $Y$ for some $R>0$. A metric $d$ on $X$ is called proper if every closed ball $B_{r}(x, d)=\{y \in X \mid d(x, y) \leq r\}$ in $X$ is compact. We denote by $\dot{B}_{r}(x, d)=\{y \in X \mid d(x, y)<r\}$ the open ball of radius $r$ centered at $x$. A metric $d$ on a group $G$ is called left invariant if $d(x, y)=d(g x, g y)$ for all $g, x, y \in G$.

Proposition 1.1. Let $d$ and $d^{\prime}$ be two proper left invariant metrics on a group $G$ which induce the same topology. Then the identity map id : $(G, d) \rightarrow\left(G, d^{\prime}\right)$ is a coarse equivalence.

Proof. Let $e \in G$ be the unit. We define

$$
\begin{aligned}
& \varrho_{1}(t)=\min \left\{d^{\prime}(e, z) \mid z \in G \backslash \dot{B}_{t}(e, d)\right\}, \\
& \varrho_{2}(t)=\max \left\{d^{\prime}(e, z) \mid z \in B_{t}(e, d)\right\} .
\end{aligned}
$$

The properness of $d$ and $d^{\prime}$ implies that $\varrho_{1}$ (and hence $\varrho_{2}$ ) tends to infinity. Let $x, y \in G$. We apply the obvious inequalities

$$
\varrho_{1}(d(e, z)) \leq d^{\prime}(e, z) \leq \varrho_{2}(d(e, z))
$$

with $z=x^{-1} y$ to obtain the required inequalities

$$
\varrho_{1}(d(x, y)) \leq d^{\prime}(x, y) \leq \varrho_{2}(d(x, y)) .
$$

Corollary 1.2. Let $\Gamma \subset G$ be a closed subgroup and suppose that $\Gamma$ and $G$ are supplied with proper left invariant metrics $d$ and $d^{\prime}$, respectively, that induce the same topology on $\Gamma$. Then the inclusion $(\Gamma, d) \rightarrow\left(G, d^{\prime}\right)$ is a coarse embedding.

Let $\Gamma$ be a group. We recall that a map $\|\cdot\|: \Gamma \rightarrow[0, \infty)$ is said to be a norm on $\Gamma$ if the following three properties hold:

(1) $\|x\|=0$ if and only if $x=e$,

(2) $\left\|x^{-1}\right\|=\|x\|$ for all $x \in \Gamma$,

(3) $\|x y\| \leq\|x\|+\|y\|$ for all $x, y \in \Gamma$.

Norms on a group $\Gamma$ are in bijective correspondence with left invariant metrics: Given a norm $\|\cdot\|$ on $\Gamma$, define a metric by $d(x, y)=\left\|x^{-1} y\right\|$, and given a left invariant metric $d$ on $\Gamma$ define $\|x\|=d(e, x)$. We call a norm proper if it is proper as a map (where $\Gamma$ has the topology induced by the associated metric). Note that proper norms correspond to proper metrics.

Definition. Let $\Gamma$ be a countable discrete group. Let $S$ be a symmetric generating set (possibly infinite), $S=S^{-1}$, for $\Gamma$. A weight function 
$w: S \rightarrow[0, \infty)$ on $S$ is any positive, proper function such that $w\left(s^{-1}\right)=w(s)$ for all $s \in S$. The properness can essentially be viewed as the requirement that $\lim w=\infty$.

Proposition 1.3. Every weight function $w$ on a countable group defines a proper norm

$$
\|x\|_{w}=\inf \left\{\sum_{i=1}^{n} w\left(s_{i}\right) \mid x=s_{1} \cdots s_{n}, s_{i} \in S\right\} .
$$

Proof. Obviously, the infimum in the above definition is a minimum. Then the conditions (1)-(3) are easy to check.

2. Asymptotic dimension of general groups. Gromov defined the asymptotic dimension of a metric space $X$ as follows $[\mathrm{Gr}]$.

Definition. We say that a metric space $X$ has asymptotic dimension $\leq n$ if, for every $d>0$, there is an $R$ and $n+1 d$-disjoint, $R$-bounded families $\mathcal{U}_{0}, \mathcal{U}_{1}, \ldots, \mathcal{U}_{n}$ of subsets of $X$ such that $\bigcup_{i=0}^{n} \mathcal{U}_{i}$ is a cover of $X$.

We say that a family $\mathcal{U}$ of subsets of $X$ is $R$-bounded if $\sup \{\operatorname{diam} U$ | $U \in \mathcal{U}\} \leq R$. Also, $\mathcal{U}$ is said to be $d$-disjoint if $d(x, y)>d$ whenever $x \in U$, $y \in V, U \in \mathcal{U}, V \in \mathcal{U}$, and $U \neq V$.

It follows from the definition that $\operatorname{asdim} X \leq \operatorname{asdim} Y$ for a subset $X \subset Y$ taken with the restricted metric.

The notion of asdim is a coarse invariant (see [Ro2]), i.e. $\operatorname{asdim} X=$ asdim $X^{\prime}$ for coarsely equivalent metric spaces. Since for a coarse embedding $f: X \rightarrow Y$ the image $f(X)$ is coarsely equivalent to $X$, we obtain $\operatorname{asdim} X \leq$ $\operatorname{asdim} Y$.

In view of Propositions 1.1 and 1.3 the invariant $\operatorname{asdim} \Gamma$ is well defined for every countable group $\Gamma$. By Corollary 1.2, asdim $\Gamma^{\prime} \leq \operatorname{asdim} \Gamma$ for any subgroup $\Gamma^{\prime} \subset \Gamma$.

The following theorem reduces the study of asymptotic dimension of countable groups to the asymptotic dimension of finitely generated groups.

TheOrem 2.1. Let $G$ be a countable group. Then

$$
\operatorname{asdim} G=\sup \operatorname{asdim} F
$$

where the supremum is taken over finitely generated subgroups $F$ of $G$.

Proof. Fix a weight function $w: G \rightarrow[0, \infty)$; let $\|\cdot\|$ and $d$ denote the induced norm and metric, respectively. If $\sup \operatorname{asdim} F=\infty$, then $\operatorname{asdim} G=\infty$, and we are finished. We will now assume $\sup \operatorname{asdim} F<\infty$. Set $m=$ $\sup \operatorname{asdim} F$.

Let $d>0$ be given. Set $T=\{g \mid w(g)<d\}$ and $F=\langle T\rangle$. By the definition of weight function, $T$ is finite and so $F$ is finitely generated. Thus, $\operatorname{asdim} F \leq m$. So there exist uniformly bounded, $d$-disjoint families 
$\mathcal{U}_{0}, \mathcal{U}_{1}, \ldots, \mathcal{U}_{m}$ of subsets of $F$ such that $\bigcup_{i} \mathcal{U}_{i}$ is a cover of $F$. Let $Z$ be a system of representatives for the partition by cosets $G / F$. For $0 \leq i \leq m$, define $\mathcal{V}_{i}=\left\{z U \mid z \in Z, U \in \mathcal{U}_{i}\right\}$.

It is easy to check that $\mathcal{V}_{i}$ is uniformly bounded for $i=0,1, \ldots, m$, and that $\bigcup_{i} \mathcal{V}_{i}$ is a cover of $G$. We check now that $\mathcal{V}_{i}$ is a $d$-disjoint family. For suppose $z U \neq z^{\prime} U^{\prime}$, where $z, z^{\prime} \in Z$ and $U, U^{\prime} \in \mathcal{U}_{i}$. Let $x \in z U$ and $y \in z^{\prime} U^{\prime}$. First suppose $z \neq z^{\prime}$. Note that $z U \subset z F$ and $z^{\prime} U^{\prime} \subset z^{\prime} F$, so that $x \in z F$ and $y \in z^{\prime} F$. Since $z \neq z^{\prime}$, we have $z F \neq z^{\prime} F$. But $x F=z F$ and $y F=z F$. So $x F \neq y F$, and hence $x^{-1} y \notin F$. This means that $x^{-1} y$ cannot be written as a product of elements of $T$, and so $d(x, y)=\left\|x^{-1} y\right\| \geq d$ by definition of $\|\cdot\|$. Now suppose $z=z^{\prime}$. So $x=z u$ and $y=z u^{\prime}$ for some $u \in U$ and $u^{\prime} \in U^{\prime}$. Since we must have $U \neq U^{\prime}$, we see that $U$ and $U^{\prime}$ are $d$-disjoint; thus, $d(x, y)=d\left(z u, z u^{\prime}\right)=d\left(u, u^{\prime}\right) \geq d$. It follows that the family $\mathcal{V}_{i}$ is $d$-disjoint.

Since $d>0$ was arbitrary, asdim $G \leq m$. Equality immediately follows.

REMARK 1 . Since every finitely generated subgroup of $\mathbb{Q}$ is cyclic, Theorem 2.1 implies in particular that $\operatorname{asdim} \mathbb{Q}=1$ (see $[\mathrm{Sm}]$ for a direct computation).

Theorem 2.1 could lead to the the following generalization.

Definition. Let $G$ be an arbitrary group. We define $\operatorname{asdim} G=\sup \{\operatorname{asdim} F \mid F \subset G$ is finitely generated $\}$.

It is immediate that $\operatorname{asdim} G \leq \operatorname{asdim} H$ whenever $G \leqslant H$.

REMARK 2. Define a coarse structure $\mathcal{E}$ on $G$ as follows: $E \in \mathcal{E}$ if and only if $\left\{x^{-1} y \mid(x, y) \in E\right\}$ is a finite set. It can be shown that the definition of asymptotic dimension above coincides with the asymptotic dimension of $G$ equipped with the coarse structure $\mathcal{E}$. It is not hard to see that all bounded sets are finite. In the case when $G$ is countable, this coarse structure is precisely the bounded coarse structure associated with a proper, left invariant metric. Also, $\mathcal{E}$ is generated by the family $\left\{\Delta_{g} \mid g \in G\right\}$, where $\Delta_{g}=\{(h, h g) \mid h \in G\}$. This shows that $\mathcal{E}$ is a natural generalization of Example 2.13 from [Ro2]. Using the terminology found in this example, $\Delta_{g}$ is the $G$-saturation of $\{(1, g)\}$, and $\mathcal{E}$ is the coarse structure generated by the $G$-saturations of the finite subsets of $G \times G$. We refer to [Ro2] for the corresponding definitions.

REMARK 3. Even for familiar (uncountable) groups, this new definition of asymptotic dimension does not agree with the asymptotic dimension obtained when the group is equipped with a left invariant, proper metric. For example, using a formula from Theorem 3.2 below, we can show that $\operatorname{asdim} \mathbb{R}=\infty$ and $\operatorname{asdim} \mathbb{R} / \mathbb{Z}=\infty$. 
Though the results stated below hold true for general discrete groups, the most important case is the case of countable groups.

We recall a notion of the $R$-stabilizer from [B-D1].

Definition. Suppose that a group $\Gamma$ acts on a metric space $X$ by isometries. Let $x_{0} \in X$. For every $R>0$ we define the $R$-stabilizer of $x_{0}$ as follows:

$$
W_{R}\left(x_{0}\right)=\left\{g \in \Gamma \mid d\left(g\left(x_{0}\right), x_{0}\right) \leq R\right\} .
$$

We extend to general groups the following theorems from [B-D2].

TheOREM 2 of [B-D2]. Suppose that a finitely generated group $\Gamma$ acts on a geodesic space $X$ by isometries. Let $x_{0} \in X$ and suppose $\operatorname{asdim} W_{R}\left(x_{0}\right) \leq n$ for all $R>0$. Then $\operatorname{asdim} \Gamma \leq n+\operatorname{asdim} X$.

Theorem 7 OF [B-D2] (Hurewicz type formula). Let $\phi: G \rightarrow H$ be a homomorphism of finitely generated groups with kernel $K$. Then

$$
\operatorname{asdim} G \leq \operatorname{asdim} H+\operatorname{asdim} K .
$$

Since at the time when [D-B2] was written, the notion of asymptotic dimension was defined only for finitely generated groups, $K$ is treated in Theorem 7 as a metric space with the word metric restricted from $G$.

TheORem 2.2. Suppose that a group $\Gamma$ acts on a geodesic space $X$ by isometries. Let $x_{0} \in X$ and suppose asdim $W_{R}\left(x_{0}\right) \leq n$ for all $R>0$. Then $\operatorname{asdim} \Gamma \leq n+\operatorname{asdim} X$.

Proof. Let $F \subset \Gamma$ be a finitely generated subgroup. Then the $R$-stabilizer with respect to $F$ is contained in the $R$-stabilizer with respect to $\Gamma$. Then by Theorem 2 of [B-D2], $\operatorname{asdim} F \leq n+\operatorname{asdim} X$. By the definition, $\operatorname{asdim} \Gamma \leq$ $n+\operatorname{asdim} X$.

Theorem 2.3 (Hurewicz type formula). Let $\phi: G \rightarrow H$ be a homomorphism of groups with kernel $K$. Then

$$
\operatorname{asdim} G \leq \operatorname{asdim} H+\operatorname{asdim} K .
$$

Proof. Let $F \subset G$ be a finitely generated subgroup and let $d^{F}$ denote the word metric on $F$. Then

$$
\operatorname{asdim} F \leq \operatorname{asdim} \phi(F)+\operatorname{asdim}\left(K \cap F,\left.d^{F}\right|_{K \cap F}\right)
$$

by Theorem 7 of [B-D2]. If $\operatorname{asdim}\left(K \cap F,\left.d^{F}\right|_{K \cap F}\right)<\infty$, then by Theorem 2.1, $\operatorname{asdim}\left(K \cap F,\left.d^{F}\right|_{K \cap F}\right)=\operatorname{asdim} F^{\prime}$ for some finitely generated group $F^{\prime} \subset K \cap F$. Then by the definition $\operatorname{asdim} \phi(F) \leq \operatorname{asdim} H$ and $\operatorname{asdim} F^{\prime} \leq \operatorname{asdim} K$. Hence $\operatorname{asdim} F \leq \operatorname{asdim} H+\operatorname{asdim} K$. The assertion now follows from the definition of $\operatorname{asdim} G$. If $\operatorname{asdim}\left(K \cap F,\left.d^{F}\right|_{K \cap F}\right)=\infty$, then by Theorem 2.1 there is a sequence $F_{n}^{\prime} \subset K \cap F$ of finitely generated subgroups with asdim $F_{n}^{\prime} \geq n$. Then $\operatorname{asdim} K=\infty$ and the inequality follows. 
3. Asymptotic dimension of solvable groups. A group $T$ is called a torsion group if every element of $T$ has a finite order.

In view of the facts that every finite group has asymptotic dimension 0 and every finitely generated torsion abelian group is finite, we obtain the following.

Lemma 3.1. $\operatorname{asdim} T=0$ for every torsion abelian group.

Theorem 3.2. Let $A$ be an abelian group. Then

$$
\operatorname{asdim} A=\operatorname{dim}_{\mathbb{Q}}(A \otimes \mathbb{Q}) .
$$

Proof. Let $\operatorname{dim}_{\mathbb{Q}}(A \otimes \mathbb{Q})=n$. By induction on $n$ we show that $\mathbb{Z}^{n} \subset A$. Consider a short exact sequence

$$
0 \rightarrow \mathbb{Z} \rightarrow A \stackrel{\psi}{\longrightarrow} A / \mathbb{Z} \rightarrow 0
$$

and tensor it by $\mathbb{Q}$ to obtain $\operatorname{dim}_{\mathbb{Q}}((A / \mathbb{Z}) \otimes \mathbb{Q})=n-1$. By the induction assumption $\mathbb{Z}^{n-1} \subset A / \mathbb{Z}$. Then a section of $\psi$ over $\mathbb{Z}^{n-1}$ together with the imbedding of the fiber $\mathbb{Z} \rightarrow A$ defines a monomorphism $\mathbb{Z}^{n} \rightarrow A$. Therefore, $\operatorname{asdim} A \geq n$.

On the other hand, since every torsion free $\mathbb{Z}$-module is flat, we obtain

$$
A / \operatorname{Tor} A=(A / \operatorname{Tor} A) \otimes \mathbb{Z} \subset(A / \operatorname{Tor} A) \otimes \mathbb{Q}=A \otimes \mathbb{Q}=\mathbb{Q}^{n} .
$$

By Remark 1 and the product theorem for asymptotic dimension [D-J] we have $\operatorname{asdim} \mathbb{Q}^{n}=n$. Hence $\operatorname{asdim}(A /$ Tor $A) \leq n$. Then by the Hurewicz type formula (Theorem 2.3) and Lemma 3.1, $\operatorname{asdim} A \leq \operatorname{asdim}(A /$ Tor $A)+$ $\operatorname{asdim}(\operatorname{Tor} A) \leq n+0$.

COROLlary 3.3. For any short exact sequence of abelian groups

$$
0 \rightarrow B \rightarrow A \rightarrow C \rightarrow 0
$$

we have

$$
\operatorname{asdim} A=\operatorname{asdim} B+\operatorname{asdim} C .
$$

In particular, $\operatorname{asdim} A \times B=\operatorname{asdim} A+\operatorname{asdim} B$.

Definition. Let $\Gamma$ be solvable and let $1=G_{0} \subset G_{1} \subset \cdots \subset G_{n}=\Gamma$ be the commutator series, $G_{i}=\left[G_{i+1}, G_{i+1}\right]$. Then the Hirsch length of $\Gamma$ is defined as

$$
h(\Gamma)=\sum \operatorname{dim}_{\mathbb{Q}}\left(\left(G_{i+1} / G_{i}\right) \otimes \mathbb{Q}\right) .
$$

TheOREm 3.4. For a solvable group $\Gamma$, asdim $\Gamma \leq h(\Gamma)$.

Proof. Apply the Hurewicz type formula and Theorem 3.2.

For a virtually polycyclic group $\Gamma$, we define the Hirsch length $h(\Gamma)$ to be $h\left(\Gamma^{\prime}\right)$, where $\Gamma^{\prime}$ is a polycyclic, normal subgroup of $\Gamma$ of finite index. It is easy to check that this Hirsch length is well defined. 
TheOREM 3.5. For a virtually polycyclic group $\Gamma$,

$$
\operatorname{asdim} \Gamma=h(\Gamma) .
$$

Proof. Since both the asymptotic dimension and the Hirsch length are left unchanged by passing from a virtually polycyclic group to a polycyclic subgroup of finite index, it suffices to prove this theorem when $\Gamma$ is polycyclic. In view of Theorem 3.4 it suffices to show the inequality asdim $\Gamma \geq h(\Gamma)$. By Theorems 3.1 and 4.28 in [Ra], there is a normal subgroup $H \subset \Gamma$ of finite index which is a cocompact lattice in a solvable simply connected Lie group $G$. Then $H$ is coarsely isomorphic to $G$ taken with an invariant metric. On the other hand, the homogeneous space $G / K$ is coarsely isomorphic to $G$ where $K$ is a maximal compact subgroup in $G$. The metric on $G / K$ can be taken to be the Hausdorff metric on the orbits $K x$. It is well known that the homogeneous space $G / K$ is homeomorphic to $\mathbb{R}^{n}$. Since every locally compact contractible (metrically) homogeneous metric space is uniformly contractible, the space $G / K$ is uniformly contractible. One can extract from the proof of Theorem 4.28 in [Ra] that $n=h(\Gamma)$. By a theorem of Roe, $H X^{n}(G / K)=H_{\mathrm{c}}^{n}(G / K)=\mathbb{Z}$ since $G / K$ is a uniformly contractible metric space, where $H X^{*}$ denotes the coarse cohomology [Ro1]. Since the coarse cohomology is a coarse invariant, $H X^{n}(\Gamma)=\mathbb{Z}$.

We show that asdim $\Gamma \geq n$. Assume that asdim $\Gamma \leq n-1$. Then $\Gamma$ admits an anti-Čech approximation by $n$-1-dimensional locally finite polyhedra [Dr], [Ro1]. This means there is a direct system of $n$-1-dimensional locally finite polyhedra $\left\{K_{i}, \phi_{i+1}^{i}\right\}$ associated with $\Gamma$ such that

$$
0 \rightarrow \lim ^{1} H_{\mathrm{c}}^{n-1}\left(K_{i}\right) \rightarrow H X^{n}(\Gamma) \rightarrow \varliminf_{\lim } H_{\mathrm{c}}^{n}\left(K_{i}\right) \rightarrow 0 .
$$

Being isomorphic to the Čech cohomology group of a compact space, every group $H_{\mathrm{c}}^{n-1}\left(K_{i}\right)$ is countable. Since the lim-one of countable groups is either 0 or uncountable $[\mathrm{Ha}]$, we obtain a contradiction: $\mathbb{Z}=\lim _{\leftarrow}^{1} H_{\mathrm{c}}^{n-1}\left(K_{i}\right)$.

\section{References}

[B-D1] G. Bell and A. Dranishnikov, On asymptotic dimension of groups, Algebr. Geom. Topol. 1 (2001), 57-71.

[B-D2] -, 一, A Hurewicz-type theorem for asymptotic dimension and applications to geometric group theory, arXiv:math.GR/0407431, 2004.

[Dr] A. Dranishnikov, Asymptotic topology, Russian Math. Surveys 55 (2000), 10851129; transl. from: Uspekhi Mat. Nauk 55 (2000), no. 6, 71-116.

[D-J] A. Dranishnikov and T. Januszkiewicz, Every Coxeter group acts amenably on a compact space, Topology Proc. 24 (1999), 135-141.

[Gr] M. Gromov, Asymptotic invariants of infinite groups, in: Geometric Group Theory, Vol. 2, Cambridge Univ. Press, 1993, 11-295. 
[Ha] A. È. Harlap, Local homology and cohomology, homological dimension, and generalized manifolds, Math. USSR-Sb. 25 (1975), 323-349; transl. from: Mat. Sb. (N.S.) 96 (138) (1975), 347-373.

[Ra] M. S. Raghunathan, Discrete Subgroups of Lie Groups, Ergeb. Math. Grenzgeb. 68, Springer, 1972.

[Ro1] J. Roe, Coarse cohomology and index theory on complete Riemannian manifolds, Mem. Amer. Math. Soc. 104 (1993), no. 497.

[Ro2] -, Lectures on Coarse Geometry, Univ. Lecture Ser. 31, Amer. Math, Soc. 2003.

[Sm] J. Smith, Asymptotic dimension of countable abelian groups, preprint, 2005.

Department of Mathematics

University of Florida

P.O. Box 118105

358 Little Hall

Gainesville, FL 32611-8105, U.S.A.

E-mail:dranish@math.ufl.edu

justins@math.ufl.edu

Received 11 April 2005;

in revised form 12 October 2005 